\title{
Decoupled Control of Position and / or Force of Tendon Driven Fingers
}

\author{
Friedrich Lange, Gabriel Quere, and Antonin Raffin
}

\begin{abstract}
In contrast to underactuated robotic hands the DLR AWIWI II hand of the David robot is fully controllable because each finger with 4 joints is actuated by 6 or 8 tendons respectively. For such fingers all joint angles (generalized positions) or joint torques (generalized forces) can be controlled independently. Usually, the specifications in joint space are converted to desired tendon forces or motor torques, which are regulated by an inner loop impedance controller. However, this conversion typically exhibits couplings between the components of the joint angle vector or the joint torque vector respectively, which arise when using the well known equations. Therefore the usual force control and position control schemes are reviewed and a generic computation of the desired tendon forces is presented. This is also done for the control of the Cartesian position and force at the finger endpoint. Thus the main contribution of the paper is the inhibition of couplings in joint space or at the Cartesian endpoint. This is demonstrated in simulations of the index finger of the DLR David hand.
\end{abstract}

\section{INTRODUCTION}

In contrast to robot arms with motors located in the joints, with robot hands there is not sufficient space for actuators within each finger joint. Therefore for most robotic hands, as e.g., [1], [2], [3], [4], [5], [6], [7], [8], the motors are placed distant from the joints. This has been a main design criterion for the DLR Hand Arm System, see [9], [10], in which the motors are located in the forearm. Then the actuation of the joints is by tendons.

However, such arrangements cause couplings, because tendons to distant joints pass by the more proximal joints. In this way it is not trivial to control the joints in a decoupled way [11], [12], [13], [14], [15], [16]. In particular it is shown in this paper that existing approaches for decoupled joint torque control, e.g. [11], offer couplings of the finger joints when being applied to joint angle control. Therefore modified controllers are proposed here that apply for both, torque control (generalized force control) and angle control (generalized position control).

In contrast to very popular underactuated setups, this paper concentrates on fully controllable systems. Then, for a finger with $n$ joints at least $n+1$ tendons have to be specified [11], [17]. Otherwise the tendons may become slack or the independent control of the joints not possible. According to the classification in [18], [15], a controllable tendon driven mechanism is assumed, which besides the number of tendons includes a nonsingular routing.

The contributions of this paper apply to all such fingers. As an example the index finger of the AWIWI II hand of the

The authors are with the Institute of Robotics and Mechatronics, German Aerospace Center (DLR), 82234 Wessling, Germany. ffriedrich.lange, gabriel.quere, antonin. raffin\}edlr.de

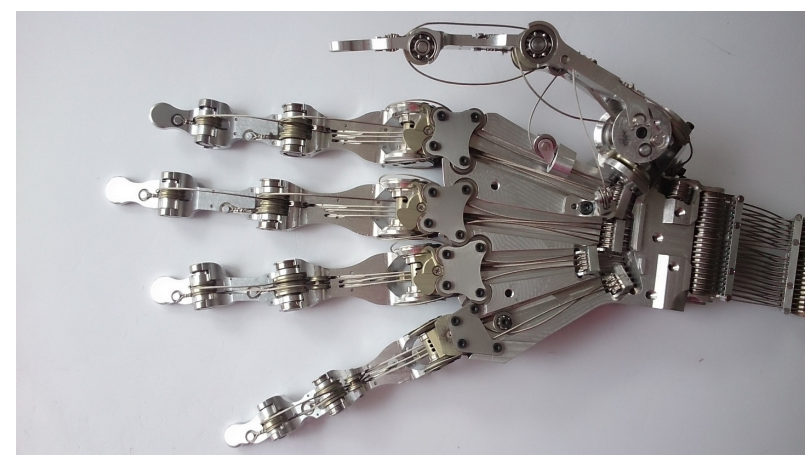

Fig. 1. DLR David hand (AWIWI II hand)

DLR David robot (formerly called DLR Hand Arm System, see Fig. 1) is considered which offers $m=8$ tendons for $n=4$ joints.

This paper subdivides control in

- the determination of the $m$ desired motor torques or tendon forces from the $n$ desired joint torques or joint angles and

- the dynamic control of the tendons by impedance control

and concentrates on the former with the emphasis on decoupling. This means that given a step of a single joint torque or joint angle, only this joint will change its state even though several tendons are involved which are connected to other joints as well.

For control, an impedance controller with the desired tendon forces as input is assumed (see Fig. 2), as implemented for the DLR David hand. Alternatively, an inner loop position control can be considered (see Fig. 3), as it is used, e.g., for the Robonaut 2 [19]. Position control is preferred whenever tendon forces cannot be measured accurately enough or whenever the motors do not allow torque control, as with step motors. For brevity it is not discussed here.

For a finger in contact, surely the joint torques have to be controlled, whereas the joint angles partially arise depending on the geometry of the touched object. On the other hand, before the contact is reached, the joint angles will be controlled exclusively and in doing so the joint torques will be zero. Thus the argumentation within this paper differs from approaches which use the same desired values for both, such that the finger speed depends on the desired gripping force.

Besides, alternatively to the joint values, the Cartesian position and force at the finger endpoint are considered, as in [20], [21]. Also for this case those desired motor torques 


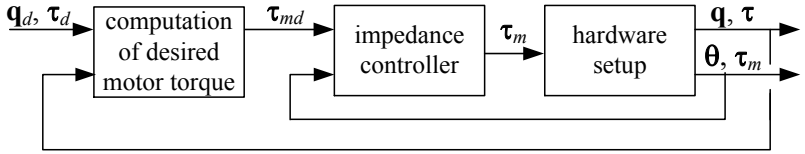

Fig. 2. Control with desired tendon forces $\mathbf{f}_{t d}$ (or desired motor torques $\boldsymbol{\tau}_{m d}$ ) as input to the inner loop.

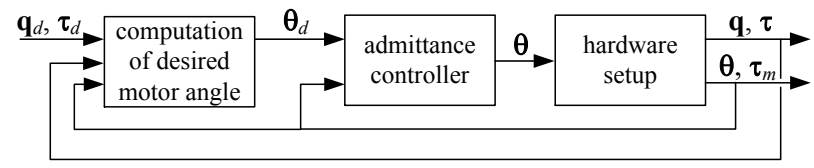

Fig. 3. Control with desired motor angles $\boldsymbol{\theta}_{d}$ (or desired motor side tendon positions $\mathbf{u}_{t d}$ ) as input to the inner loop.

or tendon forces are derived that decouple during both, force and position control. Endpoint control is important for pinch grasps in which contact to the grasped object is intended at a single contact point for each finger. Its force or position is more important than the individual joint values. For example, couplings in Cartesian position control mean that the fingers do not move in the expected way to their future contact points.

In this way the main contribution of this paper is the presentation of the desired tendon forces for different scenarios, such that the joint or Cartesian characteristics respectively are decoupled.

The paper is organized as follows: In Sect. II tendon driven systems are reviewed, introducing the notation of the setup and the control. Then Sect. III discloses couplings when controlling joint torques or joint angles and proposes modified computations of the generalized force of the motors. Sect. IV then extends the joint control methods to control of the finger endpoint. Finally simulations are reported in Sect. V.

\section{TENDON DRIVEN SYSTEMS}

In this section the setup and the notation of tendon driven fingers are reviewed, including their control.

\section{A. Setup of tendon driven fingers}

A robot finger (see Fig. 4) with $n$ joints with angle $\mathbf{q} \in \mathbb{R}^{n}$ and torque $\tau \in \mathbb{R}^{n}$ is considered. They are moved by $m$ tendons, actuated by $m$ motors with angle $\boldsymbol{\theta} \in \mathbb{R}^{m}$ and torque $\boldsymbol{\tau}_{m} \in \mathbb{R}^{m}$. At the joints the tendon position $\mathbf{x}_{t} \in \mathbb{R}^{m}$ and force $\mathbf{f}_{t} \in \mathbb{R}^{m}$ are expressed by

$$
\begin{aligned}
\mathbf{x}_{t} & =\mathbf{R q} \\
\boldsymbol{\tau} & =\mathbf{R}^{T} \mathbf{f}_{t}
\end{aligned}
$$

where $\mathbf{R} \in \mathbb{R}^{m \times n}$ is the routing matrix. This matrix contains the radii of the pulleys of the joints, where $\left|r_{i j}\right|$ is the radius of the pulley of tendon $i$ at joint $j$. The tendon routing is represented by the signs of the $r_{i j}$, where $r_{i j}>0$ means that $\Delta q_{j}>0$ increases the tendon position $x_{t i}$, whereas $r_{i j}<0$ represents the opposite routing. $r_{i j}=0$ means that tendon

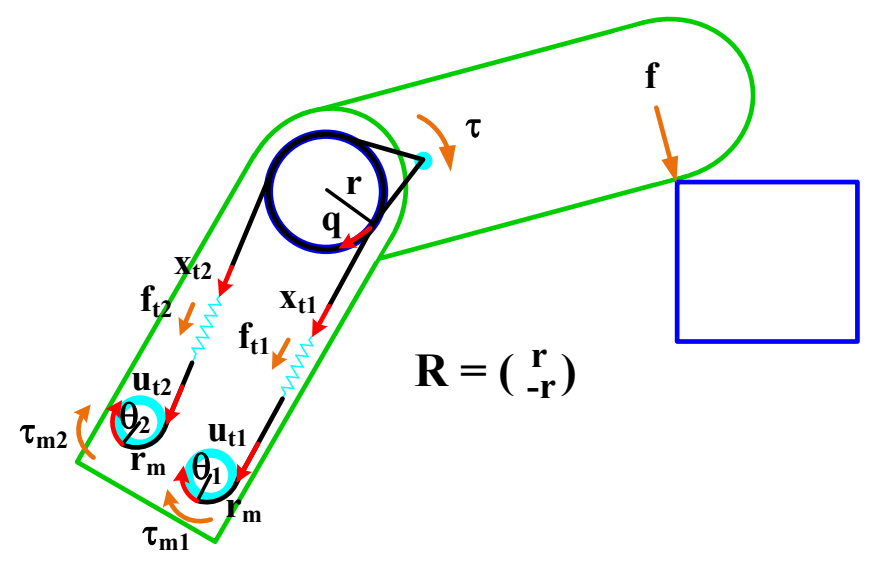

Fig. 4. Notation, shown for a single joint with two tendons.

$i$ ends before joint $j$ or that it is routed through the axis of joint $j$.

The routing matrix of most fingers of the DLR David hand is represented by

$$
\mathbf{R}=\left[\begin{array}{cccc}
R_{1} & R_{1} & 0 & 0 \\
-R_{1} & R_{1} & 0 & 0 \\
R_{1} & -R_{1} & 0 & 0 \\
-R_{1} & -R_{1} & 0 & 0 \\
R_{4} & 0 & R_{2} & 0 \\
-R_{4} & 0 & -R_{2} & 0 \\
R_{5} & 0 & R_{3} & R_{3} \\
-R_{5} & 0 & -R_{3} & -R_{3}
\end{array}\right]
$$

Column 1 represents adduction / abduction in the MCP joint whereas the other columns stand for flexion / extension in joints MCP, PIP, and DIP, respectively. In the sequel, the individual degrees of freedom (dof) represented by the columns are denoted as joints. In order to reduce the couplings, $R_{4}$ and $R_{5}$ are smaller than the other radii. The zeroes in the lower part of column 2 mean that tendons 5 to 8 are routed through the axis of joint 2 .

In contrast to [17], [22], [23] a constant routing matrix $(\mathbf{R} \neq \mathbf{R}(\mathbf{q}))$ is assumed. This means that for all joint angles all tendons are in contact to the pulleys. This is reached by an extra turn around the pulley as shown in Fig. 4.

It is assumed that a minimum tension of all tendons is guaranteed by springs between the motors and the joints. Therefore the notation has to be extended by another tendon position. Besides the tendon position at the joint $\mathbf{x}_{t}$, the tendon position at the motor is denoted by $\mathbf{u}_{t} \in \mathbb{R}^{m}$, where the same tendon force $\mathbf{f}_{t}>\left(f_{t_{\text {min }}}\right)>\mathbf{0}$ is exerted as at the joint. ${ }^{1}$

At the motors

$$
\begin{aligned}
\mathbf{u}_{t} & =\mathbf{R}_{m} \boldsymbol{\theta} \\
\boldsymbol{\tau}_{m} & =\mathbf{R}_{m} \mathbf{f}_{t}
\end{aligned}
$$

where $\mathbf{R}_{m} \in \mathbb{R}^{m \times m}$ is the diagonal matrix with the radii of the motor pulleys.

${ }^{1}$ The notation $\left(f_{t_{\text {min }}}\right)$ means a vector with identical elements. 
The relation between the two tendon positions $\mathbf{x}_{t}$ and $\mathbf{u}_{t}$ is given by

$$
\Delta \mathbf{f}_{t}=\mathbf{K}_{t}\left(\Delta \mathbf{u}_{t}-\Delta \mathbf{x}_{t}\right)
$$

where $\Delta$ denotes the difference between two values that are close together, as between sampling steps. $\mathbf{K}_{t} \in \mathbb{R}^{m \times m}$ is the diagonal matrix of the tendon stiffness with positive elements $k_{t i}$ which may depend on $\mathbf{f}_{t}$.

A pinch grasp is considered in which the finger is in contact or tries to get contact to a grasped object at a single contact point $\mathbf{x} \in \mathbb{R}^{3}$, the endpoint. There a contact force f $\in \mathbb{R}^{3}$ is exerted. For this paper it is assumed that the endpoint with respect to the finger is known and thus the endpoint Jacobian $\mathbf{J}(\mathbf{q}) \in \mathbb{R}^{3 \times n}$ is given. Then,

$$
\begin{aligned}
\Delta \mathbf{x} & =\mathbf{J} \Delta \mathbf{q} \\
\boldsymbol{\tau} & =\mathbf{J}^{T} \mathbf{f} .
\end{aligned}
$$

\section{B. Control of tendon driven fingers}

Equation (1) computes the joint-side tendon position $\mathbf{x}_{t}$ whenever the joint angles are given. However, for control, the tendon force $\mathbf{f}_{t}$ has to be known, but (2) does not allow to compute the tendon force $\mathbf{f}_{t} \in \mathbb{R}^{m}$ from the given joint torque $\tau \in \mathbb{R}^{n}$ since because of $m>n$ there are infinite solutions. [11], [15] therefore define

$$
\mathbf{f}_{t d}=\mathbf{R}^{+T} \boldsymbol{\tau}_{d}+\mathbf{f}_{t_{0}}
$$

for the specification of desired tendon forces $\mathbf{f}_{t d}$ from the desired joint torques $\tau_{d}$, using the pseudoinverse according to Appendix A.

In order to ensure that the pretension $\mathbf{f}_{t_{0}}$ does not contribute to the joint torque, i.e.

$$
\mathbf{R}^{T} \mathbf{f}_{t_{0}}=\mathbf{0}
$$

[11] specifies $\mathbf{f}_{t_{0}}$ as

$$
\mathbf{f}_{t_{0}}=\mathbf{R}_{1} \mathbf{f}_{t_{1}}
$$

with $\operatorname{rank}\left(\mathbf{R}_{1}\right)=m-n$ and $\mathbf{R}^{T} \mathbf{R}_{1}=\mathbf{0}$, e.g.

$$
\mathbf{R}_{1}=\left(\mathbf{I}_{m}-\mathbf{R R}^{+}\right)
$$

and an arbitrary $\mathbf{f}_{t_{1}}$.

As an alternative to (11) with the square matrix (12), [14] defines $\mathbf{R}_{1} \in \mathbb{R}^{m \times(m-n)}$ by unit length column vectors which are orthogonal to the columns of $\mathbf{R}$. Then an internal tension vector $\mathbf{f}_{t_{1}} \in \mathbb{R}^{m-n}$ is minimized with $\mathbf{f}_{t d}>\left(f_{t_{\text {min }}}\right)$ as constraint. In this way, with $\mathbf{R}_{1}^{T} \mathbf{R}_{1}=\mathbf{I}_{m-n}, \mathbf{R}^{T} \mathbf{R}_{1}=\mathbf{0}$, and $\left[\begin{array}{ll}\mathbf{R} & \mathbf{R}_{1}\end{array}\right]$ being invertible, (9) can be expressed as

$$
\mathbf{f}_{t d}=\left[\begin{array}{ll}
\mathbf{R} & \mathbf{R}_{1}
\end{array}\right]^{-T}\left[\begin{array}{c}
\boldsymbol{\tau}_{d} \\
\mathbf{f}_{t_{1}}
\end{array}\right] .
$$

For a given desired joint torque $\tau_{d}$, (9) or (13) can be applied directly. Instead, when controlling the joint angle, its desired value $\mathbf{q}_{d}$ specifies the desired joint torque by ${ }^{2}$

$$
\boldsymbol{\tau}_{d q}=\mathbf{K}_{P}\left(\mathbf{q}_{d}-\mathbf{q}\right)+\mathbf{K}_{D}\left(\dot{\mathbf{q}}_{d}-\dot{\mathbf{q}}\right)
$$

\footnotetext{
${ }^{2}$ In the implementation additional filtering is required.
}

computed from diagonal gain matrices $\mathbf{K}_{P}$ and $\mathbf{K}_{D} \cdot \boldsymbol{\tau}_{d q}$ is then used in (9) or (13) instead of $\boldsymbol{\tau}_{d}$. In this way the joint torque controller is switched to a joint angle controller.

\section{DECOUPLING OF JOINT SPACE CONTROL}

In this section, first it is verified whether (9) always results in decoupled control of the joints. Then (9) is modified such that this applies.

When controlling the tendon force $\mathbf{f}_{t}$, the specified joint torques will be reached whenever there is a contact such that joint torques may be applied. This is obvious from (40) in Appendix A when inserting (9) into (2), provided that $\mathbf{R}^{T} \mathbf{f}_{t_{0}}=\mathbf{0}$.

$$
\boldsymbol{\tau}=\mathbf{R}^{T}\left(\mathbf{R}^{+T} \boldsymbol{\tau}_{d}+\mathbf{f}_{t_{0}}\right)=\boldsymbol{\tau}_{d}
$$

However, for position control it is not possible to specify a joint angle or a joint trajectory directly. Even a decoupled computation of $\tau_{d q}$, as e.g. by (14), does not guarantee a decoupled motion.

For the simplified case with a diagonal inertia matrix of the motors $\mathbf{M}_{m} \in \mathbb{R}^{m \times m}$ and equal diagonal elements $r_{m}$, $m_{m}$, and $k_{t}$ of the matrices $\mathbf{R}_{m}, \mathbf{M}_{m}$, and $\mathbf{K}_{t}$, Appendix $\mathrm{C}$ shows that without contact

$$
\ddot{\mathbf{q}}=r_{m}^{2} m_{m}^{-1}\left(\mathbf{R}^{T} \mathbf{R}\right)^{-1} \boldsymbol{\tau}_{d q} .
$$

This means that a desired motion in a single component of $\mathbf{q}_{d}$ may result in actual motion of the other joints.

A way out might be

$$
\mathbf{f}_{t d}=\alpha \mathbf{R} \tau_{d q}+\mathbf{f}_{t_{0}}=\mathbf{R}^{+T}\left(\alpha \mathbf{R}^{T} \mathbf{R}\right) \boldsymbol{\tau}_{d q}+\mathbf{f}_{t_{0}}
$$

instead of (9), where according to (43) in Appendix A, both formulations of (17) are equivalent. $\alpha$ is a constant that accounts for the different orders of magnitude of $\mathbf{R}$ and $\mathbf{R}^{+T}$. $\alpha$ can also be seen as part of the controller gains $\mathbf{K}_{P}$ and $\mathbf{K}_{D}$. Then, according to Appendix C, with $\boldsymbol{\tau}=\mathbf{0}$ we get

$$
\ddot{\mathbf{q}}=r_{m}^{2} m_{m}^{-1} \alpha \boldsymbol{\tau}_{d q}
$$

instead of (16), i.e., the joints are decoupled.

Equation (17) is similar to joint-space control in [14] which is preferred there with respect to tendon-space control (13).

So with $\boldsymbol{\tau}=\mathbf{0}, \mathbf{q}_{d}$ is reached on the direct way without any coupling. However, when being in contact, typically the joint torques are specified. Then, with (17),

$$
\boldsymbol{\tau}=\mathbf{R}^{T} \mathbf{f}_{t d}=\mathbf{R}^{T}\left(\alpha \mathbf{R} \boldsymbol{\tau}_{d}+\mathbf{f}_{t_{0}}\right)=\alpha \mathbf{R}^{T} \mathbf{R} \boldsymbol{\tau}_{d},
$$

which means that the torques are coupled. In this way the specified joint torque will not be reached or at least not directly.

In this way the original approach (9) is advantageous for joint torque control, whereas the modified version (17) is preferred for joint angle control.

Both equations, (9) for generalized force control and (17) with (14) for generalized position control, can be combined by

$$
\boldsymbol{\tau}_{c}=\boldsymbol{\tau}_{d}+\alpha \mathbf{R}^{T} \mathbf{R} \boldsymbol{\tau}_{d q}
$$


which, as input to (9) and using (43), results in

$$
\mathbf{f}_{t d}=\mathbf{R}^{+T} \boldsymbol{\tau}_{d}+\alpha \mathbf{R} \boldsymbol{\tau}_{d q}+\mathbf{f}_{t_{0}} .
$$

Now there are two inputs processed, $\boldsymbol{\tau}_{d}$ and $\tau_{d q}$, instead of a single desired torque, as assumed previously. With $\mathbf{q}_{d}=$ $\mathbf{q},(21)$ is (9) and with $\boldsymbol{\tau}_{d}=\mathbf{0}$ it is (17). This allows to specify joint torques and joint angles independently, at least for different dofs.

However, even when using (21), the desired values have to be selected depending on the contact state. For example, $\tau_{d}$ has to be zero without contact. With contact the ratio of its components is constrained by the contact point. As well, with contact, $\mathbf{q}_{d}$ has to be adapted such that it is reachable. Otherwise, components of $\tau_{d}$ or $\mathbf{q}_{d}$ will not be reached properly. The specifications are easier when considering the Cartesian space (see Sect. IV).

\section{DECOUPLING OF ENDPOINT CONTROL}

Similar control approaches are possible by considering the finger endpoint instead of the joints. This could be done by transforming the desired Cartesian values to joint space and using the equations shown so far. Instead, different equations are used, which do not define $\mathbf{q}_{d}$. In other words, the joint angles arise from both, the actual and the desired endpoint position and force, such that the latter are reached faster.

Equations (7) and (44) from Appendix A result in

$$
\Delta \mathbf{x}=\mathbf{J R}^{+} \Delta \mathbf{x}_{t} .
$$

Similarly, (2) and (53) from Appendix B give

$$
\mathbf{f}=\mathbf{J}^{+T} \mathbf{R}^{T} \mathbf{f}_{t} .
$$

However, $\mathbf{f}_{t}$ cannot be computed directly from this. Thus an approach as (9) or (17) is needed, i.e.,

$$
\begin{aligned}
\mathbf{f}_{t d} & =\left(\mathbf{J}^{+T} \mathbf{R}^{T}\right)^{+} \mathbf{f}_{d}+\mathbf{f}_{t_{0}} \\
\mathbf{f}_{t d} & =\alpha_{c} \mathbf{R} \mathbf{J}^{+} \mathbf{f}_{d x}+\mathbf{f}_{t_{0}} \\
\mathbf{f}_{t d} & =\alpha_{c}\left(\mathbf{J}^{+T} \mathbf{R}^{T}\right)^{+} \mathbf{J}^{+T} \mathbf{R}^{T} \mathbf{R} \mathbf{J}^{+} \mathbf{f}_{d x}+\mathbf{f}_{t_{0}}
\end{aligned}
$$

with

$$
\mathbf{f}_{d x}=\mathbf{K}_{C P}\left(\mathbf{x}_{d}-\mathbf{x}\right)+\mathbf{K}_{C D}\left(\dot{\mathbf{x}}_{d}-\dot{\mathbf{x}}\right)
$$

using a Cartesian PD controller with diagonal gain matrices $\mathbf{K}_{C P}$ and $\mathbf{K}_{C D}$ and another scaling factor $\alpha_{c}$.

The pseudoinverse of $\mathbf{J}^{+T} \mathbf{R}^{T}$ is computed as right inverse according to Appendix B. Thus (52) in Appendix B discloses that (25) and (26) are equivalent. So the force control law (24) and the position control law (25) can be combined by

$$
\mathbf{f}_{c}=\mathbf{f}_{d}+\alpha_{c} \mathbf{J}^{+T} \mathbf{R}^{T} \mathbf{R} \mathbf{J}^{+} \mathbf{f}_{d x}
$$

which, instead of $\mathbf{f}_{d}$ is taken as input to (24).

Whenever there is contact, $\mathbf{f}_{d}$ is the desired contact force which is specified in order to reach a stable grasp. Otherwise it is zero. Depending on the task, the desired position $\mathbf{x}_{d}$ is set to $\mathbf{x}$ as soon as a stiff contact with friction is realized. Or it can then be specified in order to move the contact point. Then the value of $\alpha_{c}$ determines the desired stiffness at the finger endpoint. Thus the Cartesian desired values are more intuitive than the joint space specifications. The resulting joint configuration then arises from the setup.

With (28), $\mathbf{f}=\mathbf{f}_{d}=\mathbf{0}$, and $\mathbf{K}_{t}=k_{t} \mathbf{I}_{m}$, with (7), (44), (6), (46), (38), and (48),

$$
\ddot{\mathbf{x}}=r_{m}^{2} m_{m}^{-1} \alpha_{c} \mathbf{f}_{d x}+\partial \mathbf{J} / \partial \mathbf{x} \mathbf{J} \dot{\mathbf{q}}^{2}
$$

i.e., with small $\dot{\mathbf{q}}$ the endpoint position is decoupled.

Instead of (24) and (28), Cartesian control is also possible by (9), but then with

$$
\boldsymbol{\tau}_{c}=\mathbf{R}^{T}\left(\mathbf{J}^{+T} \mathbf{R}^{T}\right)^{+}\left(\mathbf{f}_{d}+\alpha_{c} \mathbf{J}^{+T} \mathbf{R}^{T} \mathbf{R} \mathbf{J}^{+} \mathbf{f}_{d x}\right)
$$

instead of (20). This can be derived by (2), (24), and (28). In this way (9) can be always used, with (20) and (14) or (30) and (27) respectively. Note that $\tau_{c}$ cannot be computed by $\mathbf{J}^{T} \mathbf{f}_{c}$ because control is executed by (9) and not by (24). Alternatively,

$$
\boldsymbol{\tau}_{c}=\mathbf{J}^{T} \mathbf{f}_{d}+\alpha_{c} \mathbf{R}^{T} \mathbf{R} \mathbf{J}^{+} \mathbf{f}_{d x}
$$

is found, which is identical with respect to $\mathbf{f}_{d x}$ and also converges to $\mathbf{f}_{d}$ with (9) and $\mathbf{f}_{d x}=\mathbf{0}$.

A combination of (20) and (31), e.g.

$$
\boldsymbol{\tau}_{c}=\mathbf{J}^{T} \mathbf{f}_{d}+\alpha_{c} \mathbf{R}^{T} \mathbf{R}\left(\mathbf{J}^{+} \mathbf{f}_{d x}+\beta\left(\mathbf{q}_{d}-\mathbf{q}\right)\right)
$$

may be used in order to suppress a possible null space drift of $\mathbf{q}$, where $\beta$ results in the weighting of the joint angle with respect to $\mathbf{q}_{d}=\mathbf{J}^{+} \mathbf{x}_{d}$.

\section{Simulations}

With the DLR David hand the inner loop control uses the tendon forces $\mathbf{f}_{t}$ as input. Thus (9) can directly be applied. The motor torque $\boldsymbol{\tau}_{m}$ is finally computed by an impedance law using measured values of $\mathbf{f}_{t}$ and $\boldsymbol{\theta}$, where the tendon forces are indirectly measured by the elongation of the nonlinear springs, see [24].

For a better comparability of the trajectories the experiments are simulated. For that matter the same control software as for the hardware is used, including the inner loop tendon force controller. In addition, this software features a null space shift whenever the allowed range of tendon forces of $[12 \cdots 100] \mathrm{N}$ is exceeded and equal scaling of all motor torques such that the range $[-3 \cdots 3] \mathrm{Nm}$ is not exceeded. $\alpha=100001 / \mathrm{m}^{2}, \mathbf{K}_{P}=2 \mathbf{I ~ N m}$, and $\mathbf{K}_{D}=0.0015 \mathbf{I ~ N m s}$ are selected for the controller in joint space, whereas $\alpha_{c}=$ $15, \mathbf{K}_{C P}=1333 \mathbf{I} \mathrm{N} / \mathrm{m}, \mathbf{K}_{C D}=\mathbf{I} \mathrm{Ns} / \mathrm{m}$, and $\beta=100 \mathrm{~N} / \mathrm{m}$ are used for Cartesian control. $\boldsymbol{\tau}_{d q}$ and $\mathbf{f}_{d x}$ are filtered with a time constant of $20 \mathrm{~ms}$.

The simulated measurements from the hardware are computed by

$$
\begin{aligned}
\ddot{\boldsymbol{\theta}} & =\mathbf{M}_{m}^{-1}\left(\boldsymbol{\tau}_{m}-\mathbf{R}_{m} \mathbf{f}_{t}\right) \\
\Delta \mathbf{q} & =\left(\mathbf{R}^{T} \mathbf{K}_{t} \mathbf{R}\right)^{-1} \mathbf{R}^{T} \mathbf{K}_{t} \mathbf{R}_{m} \Delta \boldsymbol{\theta}
\end{aligned}
$$

with $\tau_{m}$ as the motor torque. $\Delta$ here stands for differences with respect to the previous sampling step. Equation (34) is applied for the case in which there is no contact, i.e. $\boldsymbol{\tau}=$ $\mathbf{R}^{T} \mathbf{f}_{t}=\mathbf{0}$. See Appendix D for the derivation. 


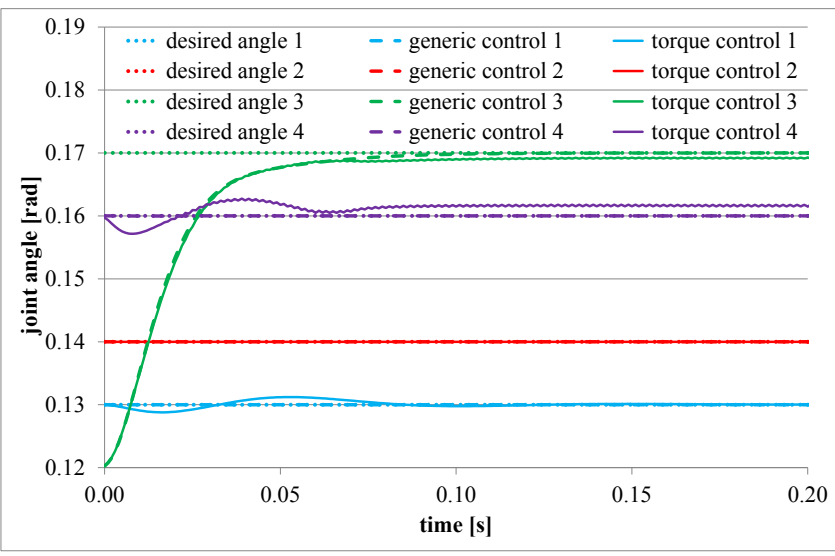

Fig. 5. Desired and actual joint angles when moving in free space: Generic control by (21) (here identical to (17)), torque control by (9) with $\boldsymbol{\tau}_{d q}$ instead of $\boldsymbol{\tau}_{d}$

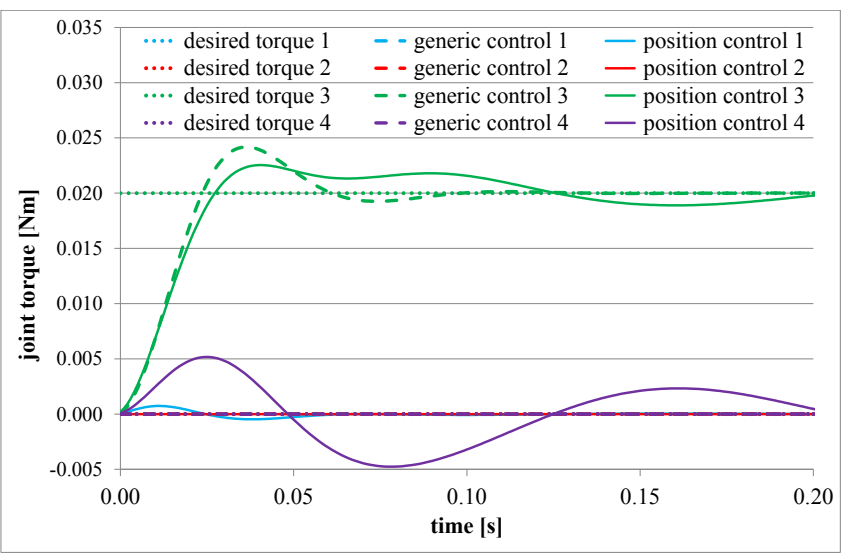

Fig. 6. Desired and actual joint torques during joint control in contact: Generic control by (21) (here identical to (9)), position control by (9) with $\boldsymbol{\tau}_{c}=\boldsymbol{\tau}+\alpha \mathbf{R}^{T} \mathbf{R}\left(\boldsymbol{\tau}_{d}-\boldsymbol{\tau}\right)$ instead of $\boldsymbol{\tau}_{d}$. (With (17) with $\boldsymbol{\tau}_{d}$ instead of $\boldsymbol{\tau}_{d q}$ not even the final joint torque is reached.)

Otherwise, we simulate that all joint angles are constrained by

$$
\mathbf{q}=\mathbf{q}_{d}
$$

which might be undue. In both cases, $\mathbf{f}_{t}$ is computed by

$$
\Delta \mathbf{f}_{t}=\mathbf{K}_{t}\left(\mathbf{R}_{m} \Delta \boldsymbol{\theta}-\mathbf{R} \Delta \mathbf{q}\right) .
$$

\section{A. Joint control}

The tests represent step responses, either of $\mathbf{q}_{d}$ in free space (Fig. 5), or of $\boldsymbol{\tau}_{d}$ in contact (Fig. 6). Each time a change of a single joint is shown because in this way couplings are seen best. A step of a single joint torque is not very realistic since, in contrast to the Cartesian case, it means that the links on both sides of the joint are fixed. This is a further argument for a simulation instead of a real experiment. In all cases the step is chosen so small that saturation effects can be almost neglected.

As expected, the generic approach of (21) works well in both configurations. Instead, in free space the original

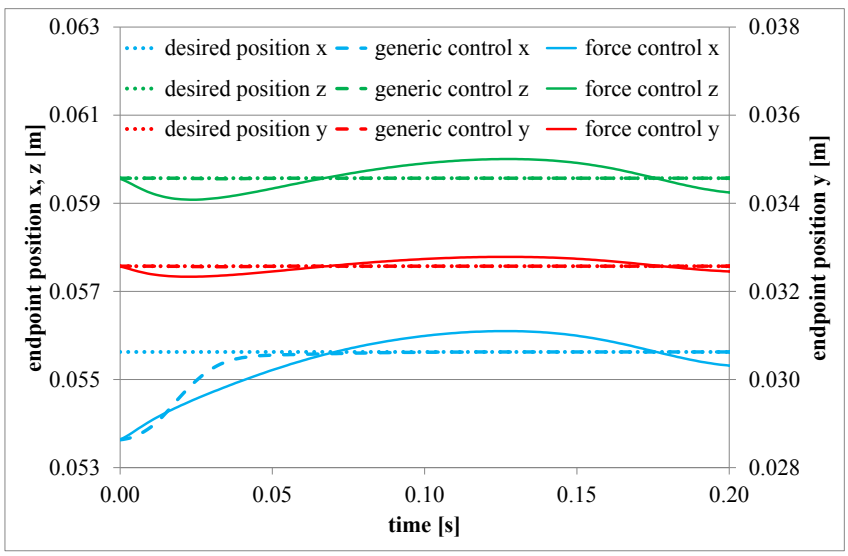

Fig. 7. Desired and actual endpoint position when moving with endpoint control in free space: Generic control by (9) with (31) (which performs as (30) or (32)), force control by (9) with $\boldsymbol{\tau}_{c}=\mathbf{J}^{T} \mathbf{f}_{d x}$.

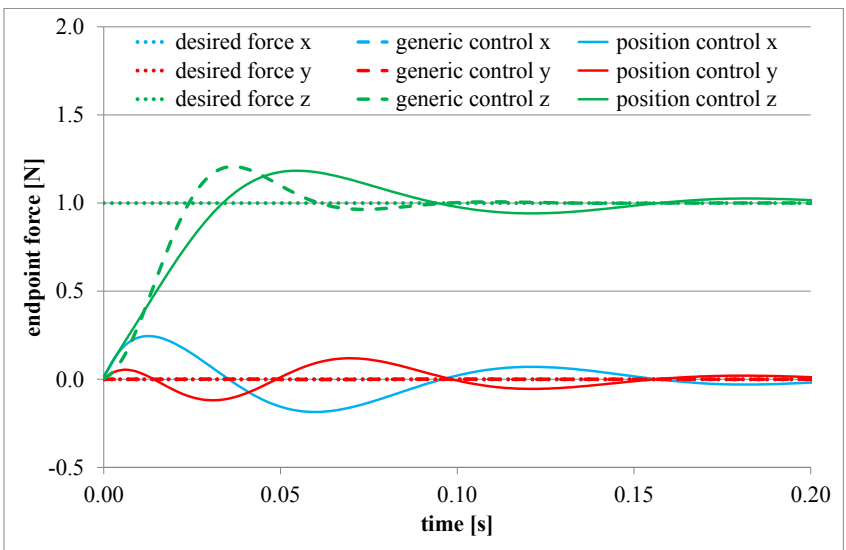

Fig. 8. Desired and actual endpoint force during endpoint control in contact: Generic control by (9) with (31) (which performs as (30) or (32)), position control by (9) with $\boldsymbol{\tau}_{c}=\boldsymbol{\tau}+\alpha_{c} \mathbf{R}^{T} \mathbf{R} \mathbf{J}^{+}\left(\mathbf{f}_{d}-\mathbf{f}\right) .\left(\boldsymbol{\tau}_{c}=\alpha_{c} \mathbf{R}^{T} \mathbf{R} \mathbf{J}^{+} \mathbf{f}_{d}\right.$ has a much too big gain.)

controller (9) shows the couplings from $\left(\mathbf{R}^{T} \mathbf{R}\right)^{-1}$, which, with a step of joint 3 , affect joint 1 and joint 4 . This justifies another approach besides (9). The experiment with contact then proves that only (17) is not suitable as well, neither with (17) and $\boldsymbol{\tau}_{d}$ instead of $\boldsymbol{\tau}_{d q}$ nor with (17) applied to the differences. In the former case even the final joint torque is not reached.

Besides the couplings, the step response is determined by the inner loop control of the tendon forces, which accounts for the time constant and the damping.

\section{B. Endpoint control}

In Cartesian space the specified steps are endpoint positions (Fig. 7) or endpoint forces (Fig. 8), also in a single component, since in this way couplings can be seen best.

Figs. 7 and 8 show that with the generic approach the Cartesian target values are reached, whereas the other approaches show a coupling. 


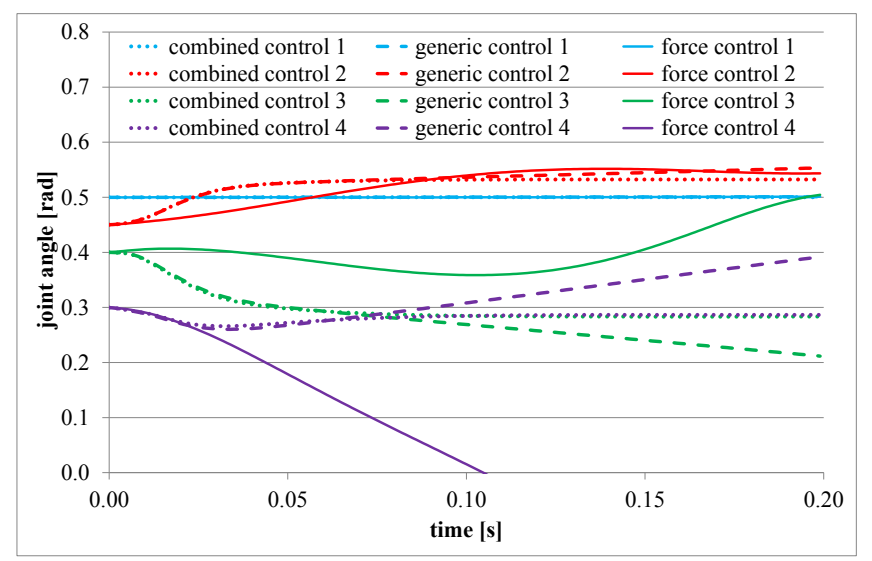

Fig. 9. Actual joint angles when moving with endpoint control in free space: Generic control by (9) with (31) (which performs as (30)), force control by (9) with $\boldsymbol{\tau}_{c}=\mathbf{J}^{T} \mathbf{f}_{d x}$, and combined control by (9) with (32).

Fig. 9 shows that apart from (32) there may be a null space drift of the joint angle during endpoint control. This is because $\mathbf{R}^{T} \mathbf{R J} \mathbf{J}_{C P} \mathbf{J}$ is not full rank. In contrast, the joint torque does not drift because it is uniquely given by (8).

\section{CONCLUSION}

The simulations verify that the generic approaches (20), (31), or (32) perform well for torque/force control as well as for angle/position control, whereas the simpler algorithms result in couplings. However, improper selection of $\mathbf{q}_{d}$ and $\boldsymbol{\tau}_{d}$ or $\mathbf{x}_{d}$ and $\mathbf{f}_{d}$ may result in an offset or a drift.

\section{APPENDIX}

\section{A. Left inverse}

For matrices with more rows than columns as the routing matrix $\mathbf{R} \in \mathbb{R}^{m \times n}$ with $m>n$, the pseudoinverse $\mathbf{R}^{+} \in \mathbb{R}^{n \times m}$ is computed as left inverse by

$$
\mathbf{R}^{+}=\left(\mathbf{R}^{T} \mathbf{R}\right)^{-1} \mathbf{R}^{T}
$$

This results in

$$
\mathbf{R}^{+} \mathbf{R}=\mathbf{I}_{n}
$$

but

$$
\mathbf{R} \mathbf{R}^{+}=\mathbf{R}\left(\mathbf{R}^{T} \mathbf{R}\right)^{-1} \mathbf{R}^{T} \neq \mathbf{I}_{m}
$$

In the same way $\mathbf{R}^{+T}$ gives

$$
\mathbf{R}^{T} \mathbf{R}^{+T}=\left(\mathbf{R}^{+} \mathbf{R}\right)^{T}=\mathbf{I}_{n},
$$

but

$$
\mathbf{R}^{+T} \mathbf{R}^{T}=\left(\mathbf{R} \mathbf{R}^{+}\right)^{T} \neq \mathbf{I}_{m}
$$

However,

$$
\mathbf{R}^{+} \mathbf{R}^{+T} \mathbf{R}^{T} \mathbf{R}=\mathbf{I}_{n}
$$

and

$$
\mathbf{R}^{+T} \mathbf{R}^{T} \mathbf{R}=\mathbf{R} .
$$

Thus (1) can be inverted to

$$
\mathbf{q}=\mathbf{R}^{+} \mathbf{x}_{t}
$$

but (2) cannot be resolved to $\mathbf{f}_{t}$.

\section{B. Right inverse}

In contrast, for matrices with less rows than columns as the endpoint Jacobian

$$
\mathbf{J} \in \mathbb{R}^{3 \times n}
$$

with $n>3$, the pseudoinverse $\mathbf{J}^{+} \in \mathbb{R}^{n \times 3}$ is computed as right inverse by

$$
\mathbf{J}^{+}=\mathbf{J}^{T}\left(\mathbf{J J}^{T}\right)^{-1} \text {. }
$$

Then,

$$
\begin{aligned}
\mathbf{J}^{+} \mathbf{J} & =\mathbf{J}^{T}\left(\mathbf{J} \mathbf{J}^{T}\right)^{-1} \mathbf{J} \neq \mathbf{I}_{n} \\
\mathbf{J J} & =\mathbf{I}_{3} \\
\mathbf{J}^{T} \mathbf{J}^{+T} & =\left(\mathbf{J}^{+} \mathbf{J}\right)^{T} \neq \mathbf{I}_{n} \\
\mathbf{J}^{+T} \mathbf{J}^{T} & =\left(\mathbf{J J}^{+}\right)^{T}=\mathbf{I}_{3} \\
\mathbf{J}^{+T} \mathbf{J}^{+} \mathbf{J} \mathbf{J}^{T} & =\mathbf{I}_{3} \\
\mathbf{J}^{+} \mathbf{J} \mathbf{J}^{T} & =\mathbf{J}^{T} .
\end{aligned}
$$

Consequently, (7) cannot be resolved to $\Delta \mathbf{q}$ but (8) can be inverted to

$$
\mathbf{f}=\mathbf{J}^{+T} \boldsymbol{\tau}
$$

\section{Analysis of joint motion}

Without contact, any specified motor torque is used for the acceleration of the motor and the link(s), where the motor inertia is dominant because it is multiplied by the square of the gear ratio. Gravity can be neglected, too. Thus $\boldsymbol{\tau}=\mathbf{0}$. Then, with (1) to (6), constant $\mathbf{K}_{t}$, and a stationary start configuration with $\mathbf{f}_{t}$, (9) results in

$$
\begin{aligned}
\mathbf{M}_{m} \ddot{\boldsymbol{\theta}} & =\mathbf{R}_{m}\left(\mathbf{f}_{t d}-\mathbf{f}_{t}\right) \\
\ddot{\mathbf{u}}_{t} & =\mathbf{R}_{m} \mathbf{M}_{m}^{-1} \mathbf{R}_{m}\left(\mathbf{f}_{t d}-\mathbf{f}_{t}\right) \\
\ddot{\mathbf{x}}_{t} & =\mathbf{R}_{m} \mathbf{M}_{m}^{-1} \mathbf{R}_{m}\left(\mathbf{f}_{t d}-\mathbf{f}_{t}\right)+\mathbf{K}_{t}^{-1} \ddot{\mathbf{f}}_{t} \\
\mathbf{R}^{T} \mathbf{K}_{t} \mathbf{R} \ddot{\mathbf{q}} & =\mathbf{R}^{T} \mathbf{K}_{t} \mathbf{R}_{m} \mathbf{M}_{m}^{-1} \mathbf{R}_{m}\left(\mathbf{f}_{t d}-\mathbf{f}_{t}\right)+\mathbf{R}^{T} \ddot{\mathbf{f}}_{t} \\
\ddot{\mathbf{q}} & =\left(\mathbf{R}^{T} \mathbf{K}_{t} \mathbf{R}\right)^{-1} \mathbf{R}^{T} \mathbf{K}_{t} \mathbf{R}_{m} \mathbf{M}_{m}^{-1} \mathbf{R}_{m}\left(\mathbf{f}_{t d}-\mathbf{f}_{t}\right) \\
\ddot{\mathbf{q}} & =\left(\mathbf{R}^{T} \mathbf{K}_{t} \mathbf{R}\right)^{-1} \mathbf{R}^{T} \mathbf{K}_{t} \mathbf{R}_{m} \mathbf{M}_{m}^{-1} \mathbf{R}_{m} \\
& \left(\mathbf{R}^{+T} \boldsymbol{\tau}_{d q}+\mathbf{f}_{t_{0}}-\mathbf{f}_{t}\right)
\end{aligned}
$$

With $\mathbf{R}_{m}, \mathbf{M}_{m}$, and $\mathbf{K}_{t}$ having equal diagonal elements $r_{m}, m_{m}$, and $k_{t}$, (37) and (2) give

$$
\begin{array}{cc}
\ddot{\mathbf{q}}= & r_{m}^{2} m_{m}^{-1}\left(\mathbf{R}^{T} \mathbf{K}_{t} \mathbf{R}\right)^{-1} \mathbf{R}^{T} \mathbf{K}_{t} \\
& \left(\mathbf{R}\left(\mathbf{R}^{T} \mathbf{R}\right)^{-1} \boldsymbol{\tau}_{d q}+\mathbf{f}_{t_{0}}-\mathbf{f}_{t}\right) \\
\ddot{\mathbf{q}}= & r_{m}^{2} m_{m}^{-1}\left(\mathbf{R}^{T} \mathbf{R}\right)^{-1}\left(\boldsymbol{\tau}_{d q}+\mathbf{R}^{T}\left(\mathbf{f}_{t_{0}}-\mathbf{f}_{t}\right)\right) \\
\ddot{\mathbf{q}}= & r_{m}^{2} m_{m}^{-1}\left(\mathbf{R}^{T} \mathbf{R}\right)^{-1} \boldsymbol{\tau}_{d q} .
\end{array}
$$

This means that joint motion is coupled by $\mathbf{R}^{T} \mathbf{R}$ which is not diagonal.

Instead, with (17) instead of (9), (55) results in

$$
\begin{aligned}
\ddot{\mathbf{q}} & =r_{m}^{2} m_{m}^{-1} \alpha\left(\mathbf{R}^{T} \mathbf{K}_{t} \mathbf{R}\right)^{-1} \mathbf{R}^{T} \mathbf{K}_{t} \mathbf{R} \boldsymbol{\tau}_{d q} \\
\ddot{\mathbf{q}} & =r_{m}^{2} m_{m}^{-1} \alpha \boldsymbol{\tau}_{d q}
\end{aligned}
$$

which is decoupled.

\section{Derivation of the equations for the simulation}

Equation (34) is derived from (6) by

$$
\begin{aligned}
\mathbf{K}_{t} \mathbf{R} \Delta \mathbf{q} & =\mathbf{K}_{t} \mathbf{R}_{m} \Delta \boldsymbol{\theta}-\Delta \mathbf{f}_{t} \\
\mathbf{R}^{T} \mathbf{K}_{t} \mathbf{R} \Delta \mathbf{q} & =\mathbf{R}^{T} \mathbf{K}_{t} \mathbf{R}_{m} \Delta \boldsymbol{\theta}-\mathbf{R}^{T} \Delta \mathbf{f}_{t},
\end{aligned}
$$

similar to the derivation of the pseudoinverse. 


\section{REFERENCES}

[1] G. Grioli, M. Catalano, E. Silvestro, S. Tono, and A. Bicchi. Adaptive synergies: an approach to the design of under-actuated robotic hands. In Proc. 2012 IEEE/RSJ Int. Conf. on Intelligent Robots and Systems(IROS), pages 1251-1256, Vilamoura, Portugal, Oct. 2012.

[2] L. Bridgwater, C. Ihrke, M. Abdallah, N. Radford, J. Rogers, S. Yayathi, R. Askew, and D. Linn. The robonaut 2 hand. In Proc. 2012 IEEE Int. Conf. on Robotics and Automation (ICRA)), pages 34253430, Saint Paul, MN, USA, May 2012.

[3] M. Ciocarlie, F. Mier Hicks, and S. Stanford. Kinetic and dimensional optimization for a tendon-driven gripper. In Proc. 2013 IEEE Int. Conf. on Robotics and Automation (ICRA), pages 2736-2743, Karlsruhe, Germany, May 2013.

[4] M. G. Catalano, G. Grioli, E. Farnioli, A. Serio, C. Piazza, and A. Bicchi. Adaptive synergies for the design and control of the pisa/iit softhand. The Int. Journal of Robotic Reasearch (IJRR), 33(5):768782, Apr. 2014.

[5] B. Belzile and L. Birglen. Stiffness analysis of double tendon underactuated fingers. In Proc. 2014 IEEE Int. Conf. on Robotics and Automation (ICRA), pages 6679-6684, Hong Kong, China, May/June 2014.

[6] C. D. Santina, G. Grioli, M. Catalano, A. Brando, and A. Bicchi. Dexterity augmentation on a synergistic hand: the pisa/iit softhand+. In Proc. 2015 IEEE/RAS Int. Conf. on Humanoid Robots (HUMANOIDS), pages 497-503, Seoul, Korea, Nov 2015.

[7] A. Mottard, T. Laliberté, and C. Gosselin. Underactuated tendondriven robotic/prosthetic hands: design issues. In Conf. on Robotics: Science and Systems (RSS XIII), Cambridge, MA, USA, July 2017.

[8] Z. Ren, C. Zhou, S. Xin, and N. Tsagarakisa. HERI hand: A quasi dexterous and powerful hand with asymmetrical finger dimensions and under actuations. In Proc. 2017 IEEE/RSJ Int. Conf. on Intelligent Robots and Systems(IROS), pages 322-328, Vancouver, BC, Canada, Sep 2017.

[9] M. Grebenstein, M. Chalon, G. Hirzinger, and R. Siegwart. Antagonistically driven finger design for the anthropomorphic DLR hand arm system. In Proc. 2010 IEEE/RAS Int. Conf. on Humanoid Robots (HUMANOIDS), pages 609-616, Nashville, TN, USA, Dec. 2010.

[10] W. Friedl, M. Chalon, J. Reinecke, and M. Grebenstein. FRCEF: the new friction reduced and coupling enhanced finger for the awiwi hand. In Proc. 2015 IEEE/RAS Int. Conf. on Humanoid Robots (HUMANOIDS), pages 140-147, Seoul, Korea, Nov 2015.

[11] H. Kobayashi, K. Hyodo, and D. Ogane. On tendon-driven robotic mechanisms with redundant tendons. The Int. Journal of Robotic Reasearch (IJRR), 17(5):561-571, May 1998.

[12] B. Siciliano and O. Khatib, editors. Handbook of Robotics. Springer, 2008.
[13] L. Birglen, T. Laliberté, and C. Gosselin. Underactuated Robotic Hands, volume 40 of Springer Tracts in Advanced Robotics. Springer, Berlin, Heidelberg, 2008

[14] M. E. Abdallah, R. Platt Jr., and C. W. Wampler. Decoupled torque control of tendon-driven fingers with tension management. The Int. Journal of Robotic Reasearch (IJRR), 32(2):247-258, 2013.

[15] R. Ozawa, H. Kobayashi, and K. Hashirii. Analysis, classification, and design of tendon-driven mechanisms. IEEE Transaction on Robotics, 30(2):396-410, April 2014

[16] M. Malvezzi, G. Gioioso, G. Salvietti, and D. Prattichizzo. SynGrasp: A MATLAB toolbox for underactuated and compliant hands. IEEE Robotics \& Automation Magazine, 22(4):52-68, Dec. 2015.

[17] J. N. A. L. Leijnse, C. W. Spoor, and R. Shatford. The minimum number of muscles to control a chain of joints with and withou tenodeses, arthrodeses, or braces - application to the human finger. Journal of Biomechanics, 38:2028-2036, 2005.

[18] R. Ozawa, K. Hashirii, and H. Kobayashi. Design and control of underactuated tendon-driven mechanisms. In Proc. 2009 IEEE Int. Conf. on Robotics and Automation (ICRA), pages 1522-1527, Kobe, Japan, May 2009.

[19] M. E. Abdallah, R. Platt Jr., B. Hargrave, and F. Permenter. Position control of tendon-driven fingers with position controlled actuators. In Proc. 2012 IEEE Int. Conf. on Robotics and Automation (ICRA)), pages 2859-2864, Saint Paul, MN, USA, May 2012.

[20] J. Inouye and F. J. Valero-Cuevas. Asymmetric routings with fewer tendons can offer both flexible endpoint stiffness control and high force-production capabilities in robotic fingers. In Proc. 2012 IEEE RAS/EMBS Int. Conf. on Biomedical Robotics and Biomechatronics (BIOMED), pages 1273-1280, Rome, Italy, June 2012.

[21] J. M. Inouye. Bio-inspired tendon-driven systems: Computational analysis, optimization, and hardware implementation. $\mathrm{PhD}$ thesis, University of Southern California, May 2012.

[22] T. Wimböck, C. Ott, A. Albu-Schäffer, A. Kugi, and G. Hirzinger. Impedance control for variable stiffnes mechanisms with nonlinear joint coupling. In Proc. 2008 IEEE/RSJ Int. Conf. on Intelligent Robots and Systems (IROS), pages 3796-3803, Nice, France, Sep. 2008.

[23] T. D. Niehues, R. J. King, A. D. Deshpande, and S. Keller. Development and validation of modeling framework for interconnected tendon networks in robotic and human fingers. In Proc. 2017 IEEE Int. Conf. on Robotics and Automation (ICRA)), pages 4181-4186, Singapore, May-June 2017.

[24] W. Friedl, M. Chalon, J. Reinecke, and M. Grebenstein. FAS: a fexible antagonistic spring element for a high performance over actuated hand. In Proc. 2011 IEEE/RSJ Int. Conf. on Intelligent Robots and Systems (IROS), pages 1366-1372, San Francisco, CA, USA, Sep 2011. 